\title{
Jacques COENEN-HUTHER, Quel avenir pour la théorie
} sociologique?

Olivier Tschannen

\section{(2) OpenEdition}

1 Journals

Édition électronique

URL : http://journals.openedition.org/ress/3152

DOI : 10.4000/ress.3152

ISBN : $1663-4446$

ISSN : $1663-4446$

Éditeur

Librairie Droz

Édition imprimée

Date de publication : 15 novembre 2015

Pagination : 262-266

ISSN : 0048-8046

Référence électronique

Olivier Tschannen, « Jacques COENEN-HUTHER, Quel avenir pour la théorie sociologique? », Revue européenne des sciences sociales [En ligne], 53-2 | 2015, mis en ligne le 15 novembre 2015, consulté le 22 septembre 2020. URL : http://journals.openedition.org/ress/3152 ; DOI : https://doi.org/10.4000/ ress.3152

Ce document a été généré automatiquement le 22 septembre 2020.

(c) Librairie Droz 


\title{
Jacques COENEN-HUTHER, Quel avenir pour la théorie sociologique?
}

\author{
Olivier Tschannen
}

\section{RÉFÉRENCE}

Jacques COENEN-HUTHER, 2015, Quel avenir pour la théorie sociologique?, Paris, L'Harmattan, « Logiques sociales », 242 p.

1 À quelles conditions la théorie sociologique peut-elle dépasser la situation de crise et de stagnation dans laquelle elle se trouve actuellement, reconquérir sa capacité à expliquer le monde contemporain et par là même retrouver sa capacité à progresser ? Telle est, à peu près, la question qui se trouve implicitement à l'origine de l'entreprise foncièrement tournée vers l'avenir de Jacques Coenen-Huther.

2 Le paradoxe de ce livre est que, pour nous parler de l'avenir, il nous replonge dans le passé de la théorie sociologique. Il y exhume des outils connus, mais un peu tombés en désuétude, et forge à partir de ces outils une série de propositions convaincantes pour une théorie résolument contemporaine, en prise directe avec la démarche empirique, et visiblement apte à décrire et à analyser les tendances les plus récentes et les plus complexes de nos sociétés.

3 La solution consiste, pour l'essentiel, à réhabiliter la notion déjà ancienne de relation. Le cœur de l'analyse sociologique n'est en effet ni l'individu et son action, ni la société comme totalité ou structure, mais bien leur relation. Et, pour ce faire, il convient de redonner une place centrale à la théorie des rôles, seule à même de fournir l'outillage théorique permettant d'analyser les ambivalences et les contradictions entre attentes de rôles incompatibles dans une société devenue hautement différenciée et segmentée.

4 La solution que nous propose Coenen-Huther n'est certes pas nouvelle, il le sait bien lui-même, puisqu'il reconnaît que la paternité de l'idée de relation revient à des auteurs aussi anciens que Simmel, Bouglé ou Dupréel (p. 205) ou encore Elias (p. 208) voire, pour citer des auteurs un peu moins anciens, Berger et Luckmann (p. 211). Son 
ambition n'est cependant pas de proposer une «nouvelle sociologie» de plus (p. 205), mais de présenter les outils qui lui semblent susceptibles de sortir la sociologie de son marasme actuel. Il ne prétend pas tant à l'originalité qu'à l'efficacité : peu lui importe que l'outillage proposé soit d'origine ancienne, l'important est qu'il fonctionne. Et il faut lui reconnaître ce mérite : tout porte à croire que la voie proposée, notamment dans les pages de conclusion qui élaborent le programme d'une sociologie relationnelle (p. 203-223), peut s'avérer fructueuse pour autant qu'on cherche à l'appliquer sérieusement.

5 Comment donc les actions individuelles se combinent-elles pour aboutir, in fine, à la création d'institutions (car c'est bien là le cœur de l'alchimie sociale qui justifie une analyse en termes de rôles) ? Coenen-Huther propose de nombreuses illustrations de ce processus, tirées de ses recherches de terrain personnelles ou des classiques de la sociologie. Parmi ces illustrations, on retiendra la métaphore de la création des chemins dans un terrain encore peu aménagé, due à l'économiste Hayek. Un premier individu cherche confusément son chemin sur le terrain encore vierge, un deuxième sera tenté de suivre sa trace, les suivants feront de même et creuseront ainsi progressivement un chemin, jusqu'au moment où le trafic sera devenu si dense qu'il appellera des formes de régulation, péages et autres mesures de signalisation, notamment de la part de la puissance étatique : «quoi de plus simple que de suivre une trace existante. [...] Ce qui s'est déjà fait devient "ce qui se fait" »(p.147). C'est ainsi qu'on passe insensiblement de la régularité à la régulation, de l'habitude à la norme. Rechercher ce qui «fait système » (p. 34) reste au fondement de la pensée sociologique. À telle enseigne que "la notion même de microsociologie est vide de sens" (p. 64). Et pourtant, dans le même temps, le social est fondamentalement "un processus » (chap. I).

6 Dans le troisième chapitre consacré à la sociologie des formes («Les formes comme constantes»), Coenen-Huther compare le milieu rural et le milieu urbain, et observe des constantes dans la manière dont se dessine la géométrie de la vie sociale dans l'un et l'autre milieu (p. 96). Il apparaît que le contexte rural est notamment marqué par la confusion des rôles. Cette forme particulière de rapports sociaux, appliquée à d'autres milieux, peut alors servir de base de raisonnement pour générer des comparaisons permettant des généralisations.

7 Le chapitre sur «L'individualisme comme méthode » (chap. IV), qui prolonge dans sa logique le premier chapitre («Le social comme processus»), attire l'attention sur les pièges que décèlent les tenants de l'individualisme méthodologique dans des démarches sociologiques apparemment anodines tant elles sont usuelles. Ainsi de la mise en relation de deux variables pour en conclure que l'une influence l'autre, ou de l'explication de la réalité sociale comme un effet de la socialisation. Dans les deux cas, ce qui manque, c'est le détour par les motivations et les raisons d'agir des acteurs. Toutefois, il convient aussi de se montrer critique envers les versions de l'individualisme méthodologique qui réduiraient ces motivations à une forme de rationalité, même limitée. Plutôt que de supposer systématiquement que les acteurs sont rationnels (même si, à l'évidence, ils le sont parfois), il est plus correct de prêter à leurs actions une forme de cohérence. Mais cette cohérence sera d'autant moins assurée que la société est plus complexe: empruntant la terminologie de Merton, CoenenHuther entend montrer que dans un environnement social hétérogène, l'acteur social est doté d'un ensemble de statuts ( status set») dont chacun est doté d'une pluralité de 
rôles ( role set »), lesquels sont pourvus d'une cohérence propre, qui est fonction des attentes des personnes concernées (p. 138). Cet ensemble complexe de statuts et de rôles explique que la construction du soi (en référence cette fois à l'héritage du pragmatisme américain) est un processus dynamique, jamais achevé.

Surgit alors une question de méthode: comment, dans cet imbroglio complexe, le sociologue peut-il discerner les motivations des acteurs? La réponse classique, héritée de Weber comme on le sait, est la suivante : par la compréhension. Mais celle-ci, affirme sans hésiter Coenen-Huther, est « une opération hasardeuse dont il n'est pas exagéré de dire qu'elle est le maillon faible de toute explication sociologique» (p.142). C'est là qu'intervient le sociologue comme être socialisé : plus il a été socialisé dans une multitude de situations elles-mêmes complexes et diverses, plus il aura de chances de percer à jour les motivations d'un acteur vivant dans un contexte pluraliste. "Le sociologue de talent est un esprit cosmopolite, capable de puiser l'inspiration dans une réserve d'expériences variées » (p. 146).

9 Le chapitre sur « Le holisme comme méthode » (chap. V), qui prolonge dans sa logique le deuxième chapitre ("La société comme système »), débute par un avertissement curieux : « le holisme n'apparaît qu'en contrepoint de l'individualisme méthodologique qui a fait l'objet d'une littérature apologétique nettement plus abondante» (p. 153). C'est certainement vrai si l'on considère l'histoire récente, celle des deux dernières décennies du siècle précédent. Mais ce n'est certainement pas vrai si l'on considère l'histoire de la sociologie dans son ensemble, notamment en France, berceau d'un holisme longtemps hégémonique avec l'école durkheimienne, qui d'ailleurs est relativement peu citée dans ce chapitre (pas plus que le structuralisme, lui aussi hégémonique plus récemment). Pour illustrer le holisme, Coenen-Huther préfère emprunter ses exemples à Tocqueville: la centralisation administrative française apparaît alors comme un trait historique illustrant la prééminence de la société comme structure globale sur les actions individuelles. À l'exemple français, le cosmopolite Coenen-Huther ajoute l'exemple russe, qu'il connait de l'intérieur, exemple d'une société qui apparait comme marquée profondément par son passé, ce qui explique "l'étonnante placidité" (p.157) de la population russe dans un contexte de dégradation radicale des conditions de vie dans les années 1990. Le "régime patrimonial " russe (notion empruntée à Weber), qui remonte au $\mathrm{xII}^{\mathrm{e}}$ siècle, se caractérise par la confusion entre souveraineté et propriété, en sorte que «la notion d'abus de pouvoir n'y a pas de sens » (p. 158), puisque le droit de propriété du sujet y est purement et simplement nié (on ne peut pas violer un droit qui n'existe pas). Par contraste, dans la France du $\mathrm{xl}^{\mathrm{e}}$ siècle telle que décrite par Tocqueville, il existait de nombreux freins à l'exercice du pouvoir : «les prérogatives de la noblesse, l'autorité des cours de justice, les droits des corporations et les privilèges des provinces. [...] Par ailleurs, la conception aristocratique de l'honneur donnait une grande force aux résistances individuelles » (p. 167-168).

On le voit, l'ouvrage que nous propose l'auteur pourrait avec le même profit servir de trame à un cours d'introduction à la sociologie (grâce à la clarté de l'écriture et aux multiples exemples très parlants) ou à la méditation de chercheurs avancés engagés dans le travail de recherche au quotidien (grâce à la pertinence et à la précision de l'argumentation). Le texte est empreint d'une tonalité discrètement autobiographique, comme lorsque l'auteur fait référence à sa " lointaine jeunesse positiviste » (p. 15). On le comprend, plus qu'une réflexion intellectuelle sur la théorie sociologique, c'est le 
bilan d'une vie de chercheur, considéré comme « engageant la totalité de sa personne " (p. 22) pour parvenir à une compréhension optimale. Coenen-Huther ne le dit pas, mais on le comprend entre les lignes : plus le sociologue avance en âge, plus il accumule des expériences sociales diverses, et par conséquent mieux il est outillé pour livrer des analyses sociologiques lucides. Dans ce métier, il existe une prime à l'expérience, surtout si celle-ci a été accumulée au cours d'une trajectoire de vie socialement bigarrée.

11 Mais au-delà du bilan personnel, c'est aussi, et surtout, le bilan de 50 ans de théorisation sociologique, depuis l'optimisme conquérant de Parsons jusqu'à ce que l'auteur, parlant de l'époque contemporaine, n'hésite pas à qualifier de « désagrégation théorique et méthodologique de la sociologie » (p. 26), en passant par les remises en cause des années 1960 et 1970 (qu'on pense à la sociologie de la sociologie de Friedrichs ou aux interrogations sur la scientificité de la sociologie consécutives à l'introduction de la notion de paradigme chez Kuhn). Bref, une pente descendante, à n'en pas douter, mais qui n'exclut pas tout espoir pour l'avenir, pour autant qu'on sache éviter les sirènes des «nouvelles sociologies" jouant avec de stériles «innovations terminologiques » (p. 28), en ayant la lucidité de leur préférer des concepts éprouvés et dotés d'une réelle fertilité heuristique, pivot d'une «conception modeste de la recherche sociologique » (p. 33).

Pour finir, il faut encore dire que l'écriture de Coenen-Huther est d'une précision et d'une sobriété remarquables. Rien qui ressemble au style jargonnant que l'on reproche si souvent aux sociologues: l'auteur est rigoureux, méthodique, simple lorsque c'est possible et prudemment complexe lorsque c'est indispensable.

Aucune recension ne saurait être complète sans critiques ou regrets. Si donc il doit y en avoir, on déplorera la place respective donnée aux parties concernant les méthodes individualiste et holiste (qui occupent un espace considérable alors qu'elles sont, au fond, désignées comme inadéquates) et aux parties concernant la théorie des rôles et la sociologie relationnelle (qui représentent la partie congrue alors qu'elles préfigurent l'avenir de la théorie sociologique). Quand bien même les chapitres sur l'individualisme et le holisme comptent maintes réflexions critiques utiles au développement d'une pensée sur l'avenir de la sociologie, il reste que ces critiques, faute d'être rassemblées en un chapitre autonome dans une architecture cohérente, perdent de leur force démonstrative. De ce fait, paradoxalement, ce livre qui nous parle de manière fort convaincante et argumentée de l'avenir de la sociologie ressemble, de par son organisation, à un état des lieux de ce que la sociologie ne devrait plus être.

\section{AUTEURS}

\section{OLIVIER TSCHANNEN}

Université de Fribourg 